\title{
sciendo
}

Int. J. of Applied Mechanics and Engineering, 2021, vol.26, No.4, pp.99-112

DOI: 10.2478/ijame-2021-0052

\section{INFLUENCE OF DIFFUSION AND IMPEDENCE PARAMETERS ON WAVE PROPAGATION IN THERMOELASTIC MEDIUM}

\author{
Sachin KAUSHAL ${ }^{*}$ \\ Department of Mathematics, School of Chemical Engineering and Physical Sciences \\ Lovely Professional University-Punjab, INDIA \\ Email: sachin_kuk@yahoo.co.in \\ Rajneesh KUMAR \\ Department of Mathematics, Kurukshetra University Kurukshetra-Haryana, INDIA \\ Kulwinder PARMAR \\ Department of Mathematics, IKG Punjab Technical University Hoshiarpur Campus, Punjab, INDIA
}

\begin{abstract}
The aim of the present paper is to study the impact of diffusion and impedance parameters on the propagation of plane waves in a thermoelastic medium for Green and Lindsay theory (G-L) and the Coupled theory (C-T) of thermoelasticity. Results are demonstrated for impedance boundary conditions and the amplitude ratios of various reflected waves against the angle of incidence are calculated numerically. The characteristics of diffusion, relaxation time and impedence parameter on amplitude ratios have been depicted graphically. Some cases of interest are also derived from the present investigation.
\end{abstract}

Key words: plane waves, amplitude ratios, diffusion, impedance parameters.

\section{Introduction}

Thermodiffusion is an extensive area of research in an elastic solid due to its many applications in the field of oil extraction. The coupling between mass diffusion, strain and fields of temperature leads to thermodiffusion. The theory of thermodiffusion in an elastic solid for a C-T model was presented by Nowacki $[1,2,3,4]$, but it implies the infinite speeds of propagation of thermoelastic waves. Sherief et al. [5] introduced the theory of generalized thermodiffusion having one relaxation time, that allows the finite speed of waves in an elastic medium. Sherief and Saleh [6] studied a half space problem in the theory of generalized thermoelastic diffusion having one relaxation time. Various authors discussed different types of problems in a thermoelastic medium [7-11].

Kumar and Kansal [12] obtained fundamental solutions and studied wave propagation problems in the theory of thermoelastic diffusion. El-Naggar et al. [13] studied the effect of the magnetic field, rotation, thermal field, initial stress and voids on the reflection of p-wave with one relaxation time. Marin et al. [14] extended the influence theorem to the generalized thermoelasticity in the context of L-S and G-L theories of thermoelasticity. Othman and Said [15] studied the effect of diffusion and internal heat source with three phase lag in the context of two-temperature theory of thermoelasticity. Saeed et al. [16] investigated the thermal effects and relaxation times in poroelastic material using finite element methods under the G-L model.

The wave propagation is part of several investigations of seismology as it provides precise results which are beneficial for economic activities like tracing of mineral ores, hydrocarbons, construction of dams, bridges, roads, the design of highways. The problem of reflection of plane waves has impedance; boundary conditions are well postulated as linear combinations of unspecifies functions and their derivatives described

\footnotetext{
${ }^{*}$ To whom correspondence should be addressed
} 
on the boundary. The most common use is that such types of problems can be observed in the field of acoustics, electromagnetism and in the area of seismology. Tiersten [17] studied the effect of a thin layer of various materials over an elastic half-space with the help of impedence boundary conditions. Malischewsky [18] investigated the propagation of Rayleigh waves with the help of impedence boundary conditions. Vinh and Hue [19] studied propagation of Rayleigh waves in an incompressible elastic half-space having impedence boundary conditions. Singh [20] investigated the problem of the reflection of elastic waves at a plane surface of an elastic half-space subjected to impedence boundary conditions.

In present investigation, the governing equations for a homogeneous isotropic thermodiffusion elastic medium are solved to find the amplitude ratios of different reflected waves in the context of the G-L theory and CT theory of thermoelasticity for impedence boundary conditions. The effects of impedance parameters and relaxation times are shown graphically for incident $\mathrm{P}$-Wave, T-Wave and MD-Wave. The new model can be applied to solve problems related to geophysics, earthquake engineering and other fields of science.

\section{Statement of the problem}

Following Sherief et al. [5] and Green and Lindsay (G-L) [21], the governing equations for isotropic, homogeneous, thermodiffusion elastic half-space (without heat sources, diffusive mass sources and body forces) in the general Cartesian coordinate system can be written as:

stress-strain-concentration-temperature relation is:

$$
t_{i j}=2 \mu e_{i j}+\delta_{i j}\left[\lambda e_{k, k}-\beta_{2}\left(1+\tau^{l} \frac{\partial}{\partial t}\right) C-\beta_{I}\left(1+\tau_{l} \frac{\partial}{\partial t}\right) T\right],
$$

equations of motion:

$$
(\lambda+\mu) \nabla(\nabla \cdot \vec{u})+\mu \nabla^{2} \vec{u}-\beta_{1}\left(1+\tau_{1} \frac{\partial}{\partial t}\right) T_{, i}-\beta_{2}\left(1+\tau^{l} \frac{\partial}{\partial t}\right) C_{, i}=\rho \ddot{u}_{i},
$$

heat conduction equation:

$$
\rho C_{E}\left(1+\tau_{0} \frac{\partial}{\partial t}\right) \frac{\partial T}{\partial t}+a T_{0}\left(1+\tau^{0} \frac{\partial}{\partial t}\right) \frac{\partial C}{\partial t}+\beta_{1} T_{0} \frac{\partial e}{\partial t}=K^{*} T_{, i i}
$$

mass diffusion equation:

$$
D \beta_{2} e_{, i i}-D b\left(1+\tau^{1} \frac{\partial}{\partial t}\right) C_{, i i}+D a\left(1+\tau_{1} \frac{\partial}{\partial t}\right) T_{, i i}+\frac{\partial C}{\partial t}=0
$$

where

$$
\begin{array}{ll}
e_{i j}=\frac{1}{2}\left(u_{i, j}+u_{j, i}\right), & (i, j=1,2,3) \\
\beta_{1}=(3 \lambda+2 \mu) \alpha_{t}, & \beta_{2}=(3 \lambda+2 \mu) \alpha_{c}
\end{array}
$$

$\lambda, \mu$ - Lame's constants, $\alpha_{t}$ - coefficient of linear thermal expansion, $\alpha_{c}$ - coefficient of diffusion expansion, $\rho$ - density, $C_{E}$ - specific heat respectively, $K^{*}$ - thermal conductivity, $T$ - temperature distribution, $t_{i j}-$ 
components of stress tensor, $e_{i j}$ - components of stain tensor, $t-$ time, $\delta_{i j}-$ Kronecker delta, $\tau_{0}, \tau_{1}-$ thermal relaxation times, $\tau^{0}, \tau^{l}$ - diffusion relaxation times, $C$ - concentration, $u_{i}$ - displacement component, $a, b$, $D$ - thermodiffusion constants, $T_{0}$ - reference temperature.

For the G-L theory

$$
\tau^{l} \geq \tau^{0} \geq 0, \quad \tau_{1} \geq \tau_{0} \geq 0
$$

for the C-T theory

$$
\tau^{l}=\tau^{0}=\tau_{1}=\tau_{0}=0
$$

\section{Formulation and solution of the problem}

We consider an isotropic, homogeneous thermally conducting diffusion elastic half-space at temperature $T_{0}$. Consider $x_{3}=0$ as the origin on the surface as well as the $x_{3}$-axis pointing normally into the medium (Fig.1.). We consider the thermoelastic plane wave in the $x_{1} x_{3}$-plane having a wavefront parallel to the $x_{2}$-axis and therefore field variables depend only on $x_{1}, x_{3}$, and $t$.

For a two dimensional problem, we take

$$
\vec{u}=\left(u_{1}, 0, u_{3}\right) \text {. }
$$

To facilitate the solution, the following dimensionless quantities are introduced:

$$
\begin{aligned}
& x_{i}^{\prime}=\frac{\omega_{1}}{c_{1}} x_{i}, \quad u_{i}^{\prime}=\frac{\rho c_{1} \omega_{1}}{\beta_{1} T_{0}} u_{i}, \quad t_{3 i}^{\prime}=\frac{1}{\beta_{1} T_{0}} t_{3 i}, \quad C^{\prime}=\frac{\beta_{2}}{\rho c_{1}^{2}} C, \\
& T^{\prime}=\frac{\beta_{1}}{\rho c_{1}^{2}} T, \quad\left(\tau_{0}^{\prime}, \tau_{1}^{\prime}, \tau^{0^{\prime}}, \tau^{l^{\prime}}, t^{\prime}\right)=\omega_{1}\left(\tau_{0}, \tau_{1}, \tau^{0}, \tau^{1}, t\right), \quad(i=1,3)
\end{aligned}
$$

where

$$
c_{1}^{2}=\left(\frac{\lambda+2 \mu}{\rho}\right) \text { and } \omega_{1}^{2}=\left(\frac{\rho C_{E} c_{1}^{2}}{K^{*}}\right) .
$$

The expression relating displacement components $u_{1}\left(x_{1}, x_{3}, t\right)$ and $u_{3}\left(x_{1}, x_{3}, t\right)$ to the scalar potential functions $q\left(x_{1}, x_{3}, s\right)$ and $\psi\left(x_{1}, x_{3}, s\right)$ in a dimensionless form are given by

$$
u_{1}=\frac{\partial q}{\partial x_{1}}-\frac{\partial \psi}{\partial x_{3}}, \quad u_{3}=\frac{\partial q}{\partial x_{3}}+\frac{\partial \psi}{\partial x_{1}}
$$

Assuming the harmonic motion, we can write

$$
(q, T, C, \psi)=(\tilde{q}, \tilde{T}, \tilde{C}, \tilde{\psi}) e^{i \omega t}
$$


where $\omega$ is known as angular frequency and $t$ is the time. Making use of Eqs (3.1)-(3.4) in Eqs (2.2)-(2.4) (suppressing the dashes and bars for ease), we obtain the following equations

$$
\begin{aligned}
& {\left[\left(1+a_{1}\right) \nabla^{2}+\omega^{2}\right] q-a_{2}\left[1+\tau_{1} i \omega\right] T-a_{2}\left[1+\tau^{l} i \omega\right] C=0,} \\
& a_{3} i \omega \nabla^{2} q+a_{2}\left[\left(1+\tau_{0} i \omega\right) i \omega-\nabla^{2}\right] T-a_{4}\left[\left(1+\tau^{0} i \omega\right) i \omega\right] C=0, \\
& a_{5} \nabla^{4} q+a_{6}\left[\left(1+\tau_{1} i \omega\right) \nabla^{2}\right] T+\left[a_{7} i \omega-a_{8}\left(1+\tau^{l} i \omega\right) \nabla^{2}\right] C=0, \\
& {\left[a_{1} \nabla^{2}+\omega^{2}\right] \psi=0}
\end{aligned}
$$

where the values of $a_{i}$ for $i=1-8$ are given in Appendix-I.

Consider a plane wave at $\left(x_{3}=0\right)$ propagating through the thermoelastic diffusion solid half space. Against each incident wave, one gets a reflected longitudinal displacement wave (P-Wave), thermal wave (TWave), mass diffusive wave (MD-Wave), shear wave (SV-Wave) as represented in Fig.1.

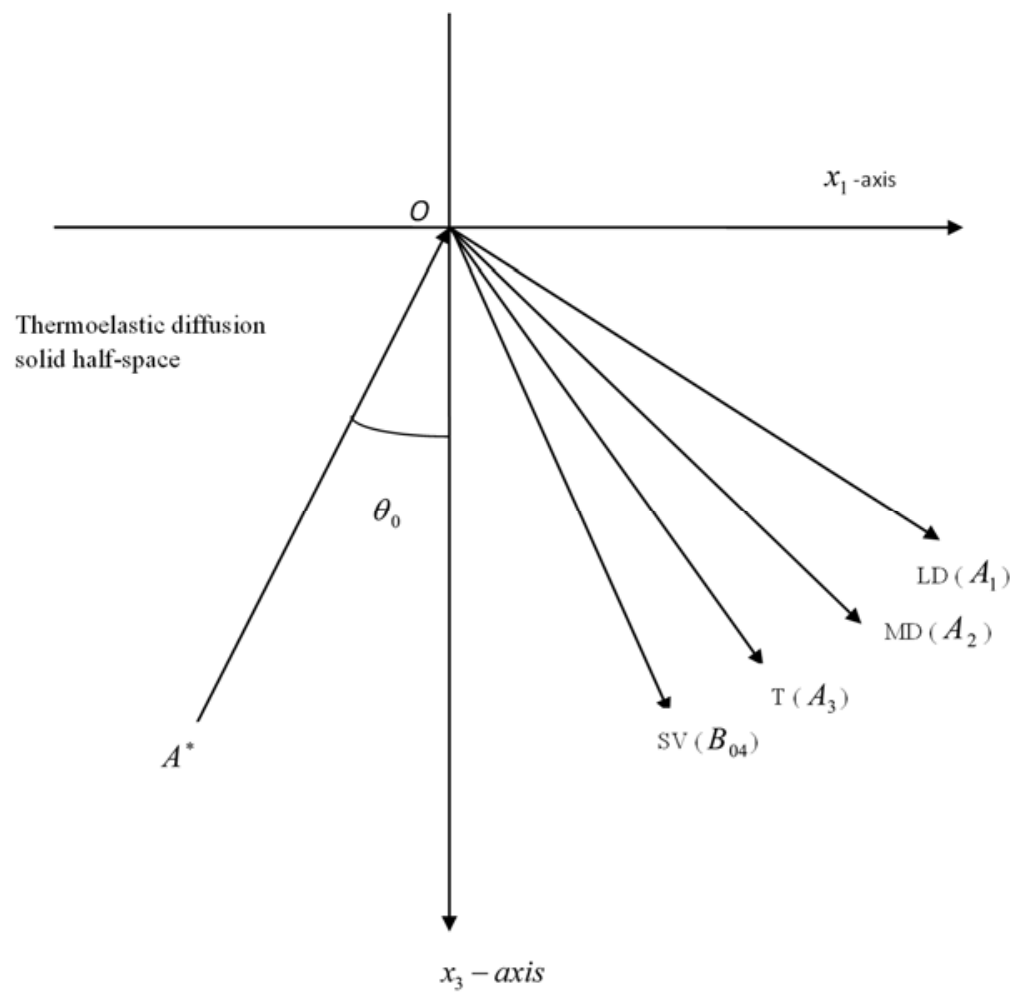

Fig.1. Geometry of the problem.

The solution of the equations in (3.5) is assumed as

$$
(q, \psi, T, C)=\left(q_{0}, \psi_{0}, T_{0}, C_{0}\right) e^{i k\left(x_{1} \sin \theta-x_{3} \cos \theta+v t\right)}
$$


where $k$ is the wave number, $q_{0}, \psi_{0}, T_{0}, C_{0}$ are constants representing the coefficients of the wave amplitude. Inserting the Eq.(3.6) in the set of equations given by Eq.(3.5), yields

$$
\begin{aligned}
& v^{6}-F v^{4}+G v^{2}-H=0, \\
& v^{2}-a_{1}=0
\end{aligned}
$$

where the values of $F, G$ and $H$ are given in Appendix-II and $v=\frac{\omega}{k}$ is the velocity of waves; $v_{1}, v_{2}$ and $v_{3}$ are the velocities of the waves, namely P-Wave, T-Wave, MD-Wave and $v_{4}$ is the velocity of the SV-Wave.

\section{Boundary conditions}

At surface $x_{3}=0$, the boundary conditions are

(i)

(ii)

$$
t_{33}+\omega Z_{1} u_{3}=0
$$

(iii)

$$
t_{31}+\omega Z_{1} u_{1}=0
$$

(iv)

$$
\frac{\partial T}{\partial x_{3}}=0
$$

$$
\frac{\partial C}{\partial x_{3}}=0
$$

where $Z_{1}$ and $Z_{2}$ are known as impedance parameters. The traction free boundary conditions can be obtained by setting $Z_{1}=Z_{2}=0$.

We assume that the values of $q, T, C$ and $\psi$ satisfy the boundary conditions (4.1) as

$$
\begin{aligned}
& {[q, T, C]=\sum\left(1, d_{l}, f_{l}\right)\left(A_{0 l} e^{i k_{l}\left(x_{1} \sin \theta_{0}-x_{3} \cos \theta_{0}\right)+i \omega t}+A_{l} e^{i k_{l}\left(x_{l} \sin \theta_{0}-x_{3} \cos \theta_{0}\right)+i \omega t}\right),} \\
& \psi=B_{04} e^{i k_{4}\left(x_{1} \sin \theta_{0}-x_{3} \cos \theta_{0}\right)+i \omega t}+B_{4} e^{i k_{4}\left(x_{1} \sin \theta_{0}-x_{3} \cos \theta_{0}\right)+i \omega t}, \quad l=1-3
\end{aligned}
$$

where the values of $d_{l}$ and $f_{l}$ are given in Appendix-III. $A_{0 l}$ are the amplitudes of various incident waves, namely P-Wave, T-Wave, MD-Wave and $B_{04}$ is the amplitude of the incident SV-Wave. $A_{l}$ are corresponding amplitudes of the reflected waves respectively and $B_{4}$ is the amplitude of the reflected SV-Wave.

Snell's law is given as

$$
\frac{\sin \theta_{0}}{v_{0}}=\frac{\sin \theta_{1}}{v_{1}}=\frac{\sin \theta_{2}}{v_{2}}=\frac{\sin \theta_{3}}{v_{3}}=\frac{\sin \theta_{4}}{v_{4}}
$$

where

$$
k_{1} v_{1}=k_{2} v_{2}=k_{3} v_{3}=k_{4} v_{4}=\omega_{1} \quad \text { at } \quad z=0,
$$

and 


$$
v_{0}= \begin{cases}v_{1}, & \text { for incident } L D-\text { wave } \\ v_{2}, & \text { for incident } T-\text { wave } \\ v_{3}, & \text { for incident } M D-\text { wave } \\ v_{4}, & \text { for incident } S V-\text { wave }\end{cases}
$$

Using the potentials as defined by Eq.(3.3) in the boundary conditions (4.1) and with the help of Eqs (4.2)(4.5), we get a system of homogeneous equations which can be written as

$$
\sum_{i, j=1}^{4} a_{i j} R_{j}=Y_{j}
$$

where $a_{i j}$ are defined in Appendix-IV.

Considering the phase of the reflected waves one can easily write using Eqs (4.2)-(4.3)

$$
\frac{\cos \theta_{j}}{v_{j}}=\left[\left(\frac{v_{0}}{v_{j}}\right)^{2}-\sin ^{2} \theta_{0}\right]^{\frac{1}{2}} .
$$

Following Schoenberg [22], if we write

$$
\begin{aligned}
& \frac{\cos \theta_{j}}{v_{j}}=\frac{\cos \theta_{j}{ }^{\prime}}{v_{j}{ }^{\prime}}+i \frac{c_{j}}{2 \pi v_{0}} \quad(j=1,2,3), \\
& \frac{\cos \theta_{j}{ }^{\prime}}{v_{j}{ }^{\prime}}=\frac{1}{v_{0}} \operatorname{Re}\left\{\left[\left(\frac{v_{0}}{v_{j}}\right)^{2}-\sin ^{2} \theta_{0}\right]^{\frac{1}{2}}\right\}+c_{j}=2 \pi I_{m}\left[\left(\frac{v_{0}}{v_{j}}\right)^{2}-\sin ^{2} \theta_{0}\right]^{\frac{1}{2}}
\end{aligned}
$$

where $v_{j}{ }^{\prime}$ the real phase speed and $\theta_{j}{ }^{\prime}$, the angle of reflection is given by

$$
\frac{v_{j}{ }^{\prime}}{v_{0}}=\frac{\sin \theta_{j}{ }^{\prime}}{\sin \theta_{0}}\left[\sin ^{2} \theta_{0}+\left[\operatorname{Re}\left(\left(v_{0} v_{4}\right)^{2}-\sin ^{2} \theta_{0}\right)^{\frac{1}{2}}\right]^{2}\right]^{\frac{-1}{2}}
$$

and $c_{j}$ is the attenuation in a depth and equals the wavelength of particular incident wave, i.e. $\left(2 \pi v_{0}\right) / \omega$.

\section{Special case}

Neglecting diffusion effect $a=b=D=0$ : we have obtained the corresponding amplitude ratio for the generalized theory of thermoelasticity for the incident P-Wave, T-Wave and SV-Wave for the thermoelastic half space with the following changed values: 


$$
\begin{aligned}
& {\left[\left(1+a_{1}\right) \nabla^{2}+\omega^{2}\right] q-a_{2} T=0, \quad a_{3} i \omega \nabla^{2} q+a_{2}\left[\left(1+\tau_{0} i \omega\right) i \omega-\nabla^{2}\right] T=0,} \\
& {\left[a_{1} \nabla^{2}+\omega^{2}\right] \psi=0}
\end{aligned}
$$

where

$$
d_{l}=\frac{a_{3} \tau_{g}-v_{l}}{\tau_{g} v_{l}^{2}-1}, \quad l=1,2
$$

\section{Numerical results and discussion}

Following Sherief and Saleh [6], the values of the relevant parameters for numerical computations are taken as:

$$
\begin{aligned}
& \mu=3.86 \times 10^{10} \mathrm{Kgm}^{-1} \mathrm{~s}^{-2}, \quad \lambda=7.76 \times 10^{10} \mathrm{Kgm}^{-1} \mathrm{~s}^{-2}, \\
& T_{0}=293 \mathrm{~K}, \quad \rho=8954 \mathrm{Kgm}^{-3} \mathrm{~K}^{*}=1.0 \times 10^{10} \mathrm{Wm}^{-1} \mathrm{~K}^{-1}, \\
& C_{E}=383.1 \times 10^{3} \mathrm{~J} \mathrm{Kg}^{-1} \mathrm{deg}^{-1} .
\end{aligned}
$$

Following Thomas [23], diffusion parameters are:

$$
\begin{aligned}
& \alpha_{t}=1.78 \times 10^{=5} \mathrm{~K}^{-1}, \quad \alpha_{c}=1.98 \times 10^{-4} \mathrm{~m}^{-3} \mathrm{Kg}^{-1}, \quad b=0.9 \times 10^{6} \mathrm{~m}^{5} \mathrm{Kg}^{-1} \mathrm{~s}^{-1} \\
& a=1.2 \times 10^{4} \mathrm{~m}^{2} \mathrm{~s}^{-2} \mathrm{~K}^{-1}, \quad D=0.85 \times 10^{-8} \mathrm{Kg} \mathrm{m}^{-3}
\end{aligned}
$$

The relaxation times are taken as:

$$
\tau_{0}=0.2 s, \quad \tau_{1}=0.4 s, \quad \tau^{0}=0.1 s, \quad \tau^{l}=0.3 s
$$

The variations of amplitude ratios for plane waves in the thermodiffusion elastic half-space along with impedance conditions are shown graphically in Figs 2-13 with the angle of incidence $0^{0} \leq \theta_{0} \leq 90^{\circ}$ for the incident P-Wave, T-Wave and MD-Wave. The solid line and dashed line correspond to the case of the coupled theory of thermoelasticity for impedance parameter $\left(Z_{1}=10\right.$ and $\left.Z_{2}=10\right)$ and non-impedance parameter $\left(Z_{1}=0\right.$ and $\left.Z_{2}=0\right)$, whereas the solid line with center symbol 'triangle' and the dashed line with center symbol 'diamond' represent the case of the G-L theory of thermoelasticity with impedance parameter $\left(Z_{1}=10\right.$ and $\left.Z_{2}=10\right)$, and non-impedance parameter $\left(Z_{1}=0\right.$ and $\left.Z_{2}=0\right)$.

\subsection{Incident P-Wave:}

Figure 2 depicts the value of the amplitude ratio $\left|R_{l}\right|$, it is observed that initially the value of $\left|R_{l}\right|$ increases in the range $0^{0} \leq \theta_{0} \leq 18^{0}$ and decreases in the remaining range. It is also noticed that the values of $\left|R_{l}\right|$ for CTNI remain smaller than the values for CTI whereas trends are opposite for the GL theory in the whole range. 


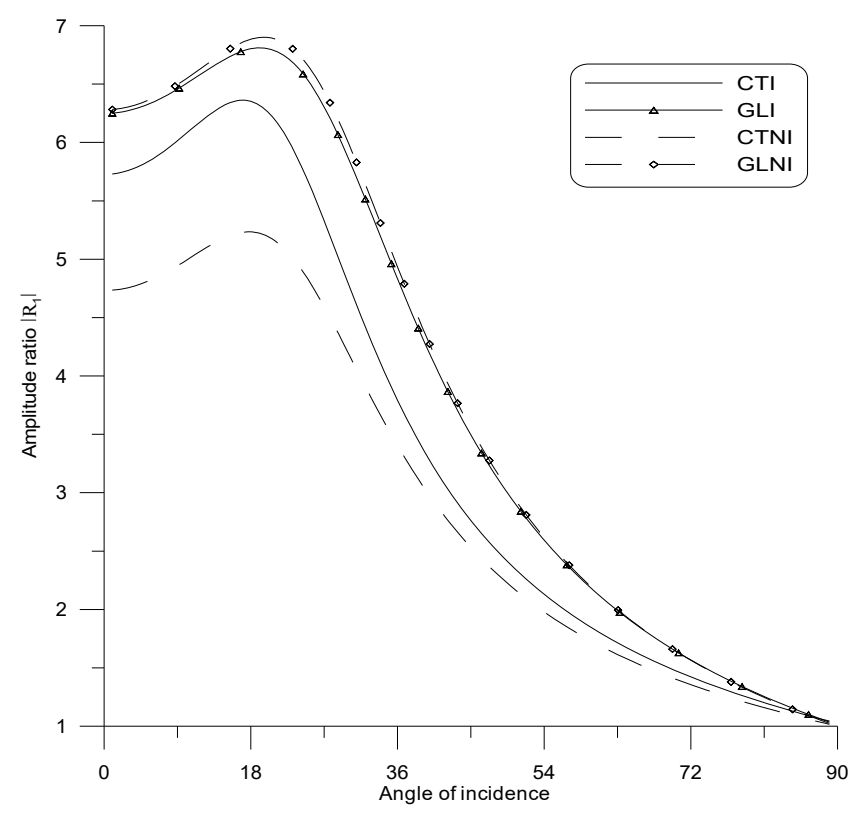

Fig.2. Variation of the amplitude ratio $\left|\mathrm{R}_{1}\right|$ for P-Wave.

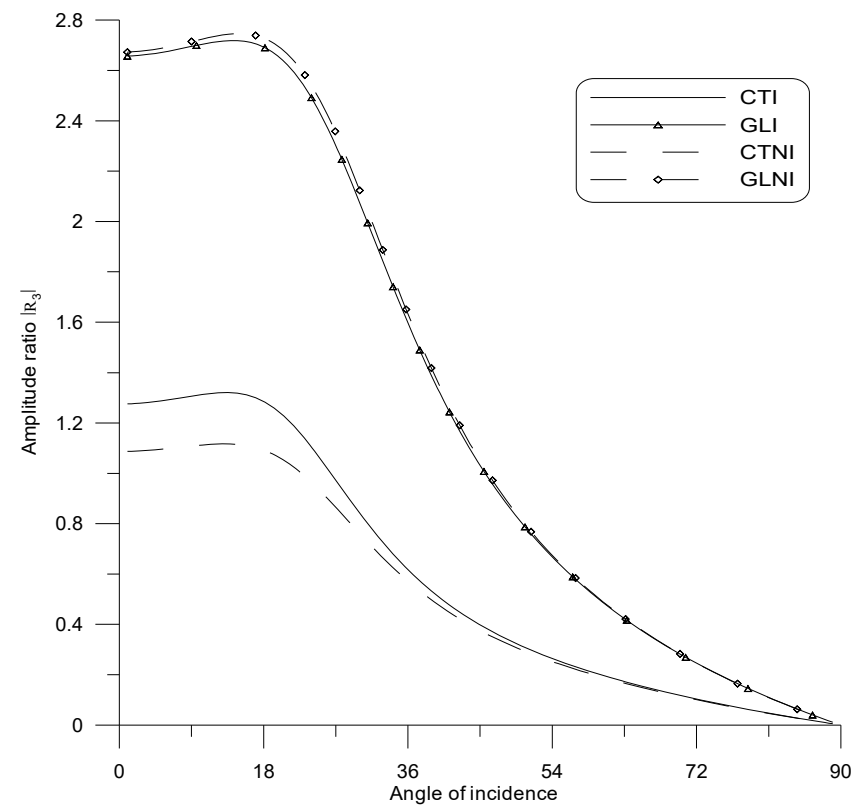

Fig.4. Variation of the amplitude ratio $\left|\mathrm{R}_{3}\right|$ for P-Wave

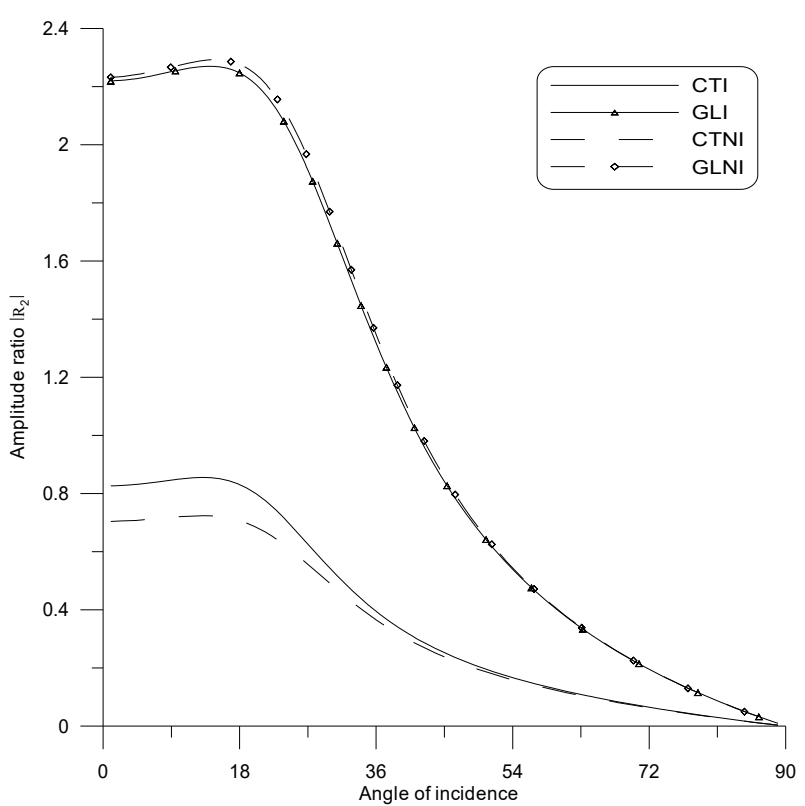

Fig.3. Variation of the amplitude ratio $\left|\mathrm{R}_{2}\right|$ for P-Wave.

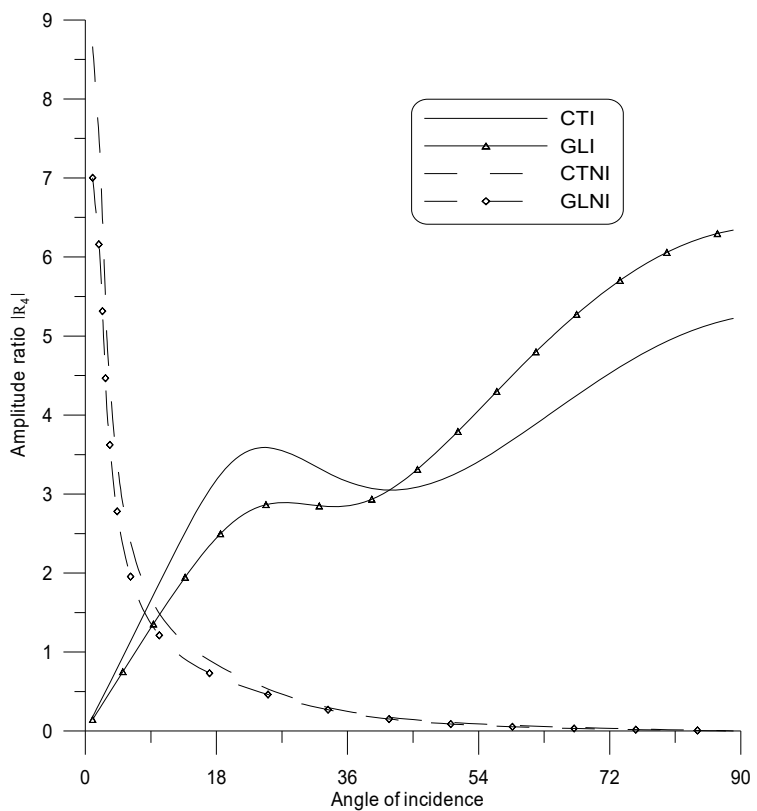

Fig.5. Variation of the amplitude ratio $\left|\mathrm{R}_{4}\right|$ for P-Wave

Figure 3 is a plot of the amplitude ratio $\left|R_{2}\right|$. It is noticed that the trend of curves for CTI is similar to CTNI in the entire range with a significant difference in their values, which reveals the impact of the impedance parameters. The amplitude ratio $\left|R_{2}\right|$ for GLI and GLNI is smaller than that observed for CTI and CTNI, which reveals the impact of the relaxation time. 
Figure 4 shows the variation of the amplitude ratio of $\left|R_{3}\right|$ along with the angle of incidence. It is observed that the trend of variation of the amplitude ratio $\left|R_{3}\right|$ is similar to that observed for $\left|R_{2}\right|$ with significant difference in their values.

Figure 5 depicts that the value of the amplitude ratio $\left|R_{4}\right|$ for the CT-theory and GL-theory in the case of $Z_{1}=10$ and $Z_{2}=10$ it increases in the range $0^{\circ} \leq \theta_{0} \leq 27^{\circ}$ and $45^{\circ} \leq \theta_{0} \leq 90^{\circ}$ whereas trends for the CTtheory and GL-theory in case of $Z_{1}=0$ and $Z_{2}=0$ are opposite in nature. The value of amplitude ratio for CTNI and GLNI decreases in the entire range and approaches zero as the value of $\theta_{0}$ increases.

\subsection{Incident T-Wave}

Figure 6 shows that the value of the amplitude ratio $\left|R_{l}\right|$ for the CT-theory and GL-theory increases in the range $0^{0} \leq \theta_{0} \leq 32^{\circ}$ and then decreases and approaches zero in the remaining range. Also it is observed that CTI and CTNI have a steeper slope as compared to GLI and GLNI.

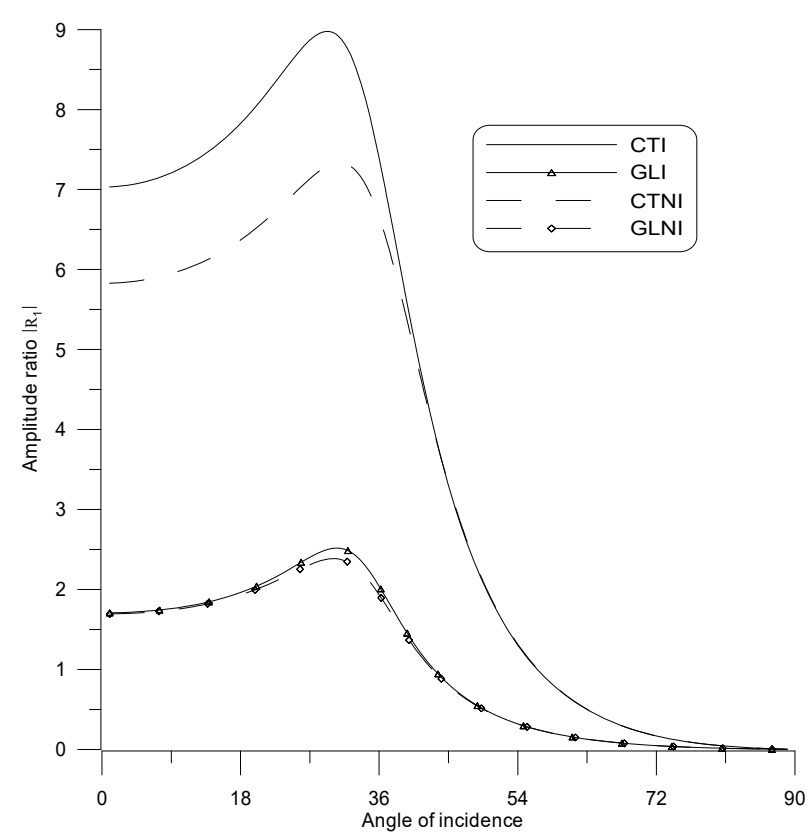

Fig.6. Variation of the amplitude ratio $\left|\mathrm{R}_{1}\right|$ for T-Wave.

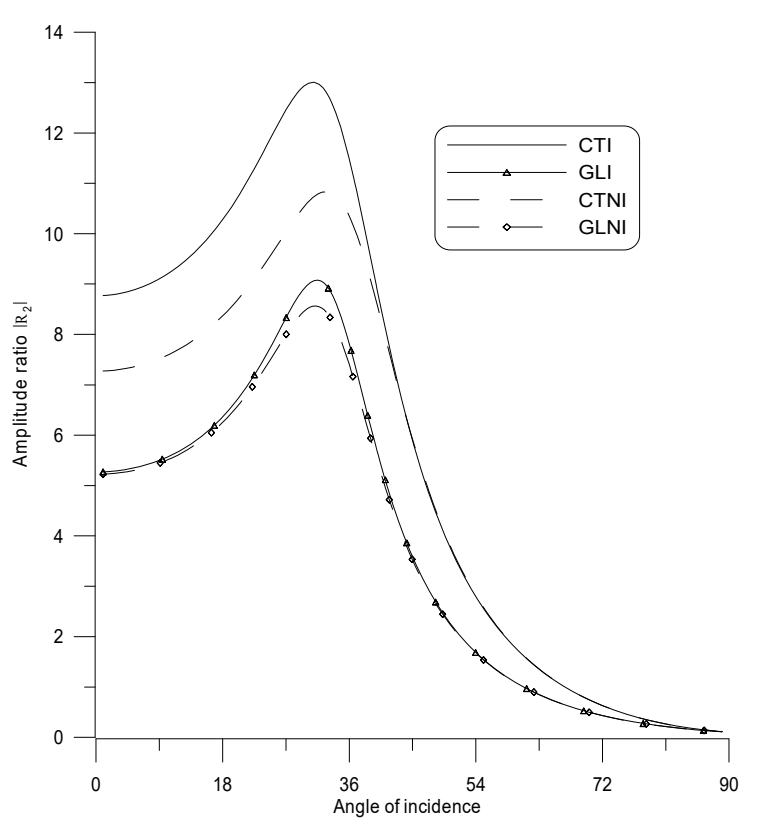

Fig.7. Variation of the amplitude ratio $\left|\mathrm{R}_{2}\right|$ for T-Wave.

Figure 7 shows the amplitude ratio $\left|R_{2}\right|$. It is observed that for the GL-theory with impedance and non-impedance parameters it is smaller than that observed for the CT-theory which shows the impact of the relaxation time.

Figure 8 is a plot of the amplitude ratio of $\left|R_{3}\right|$ along with the angle of incidence. It is noticed that the trend of variation of the amplitude ratio $\left|R_{3}\right|$ is similar to that observed for $\left|R_{2}\right|$ with notable differences in values.

Figure 9 depicts that trend for the $\left|R_{4}\right|$.It is observed that for GLI is similar to CTI as both increase in the range $0^{0} \leq \theta_{0} \leq 27^{0}$ and the trend is opposite in the remaining range and with the increase in $\theta_{0}$, the values tend to zero. Also, it is noticed that there is an overall decrease in the trend in curves for GLNI and CTNI in the whole range. 


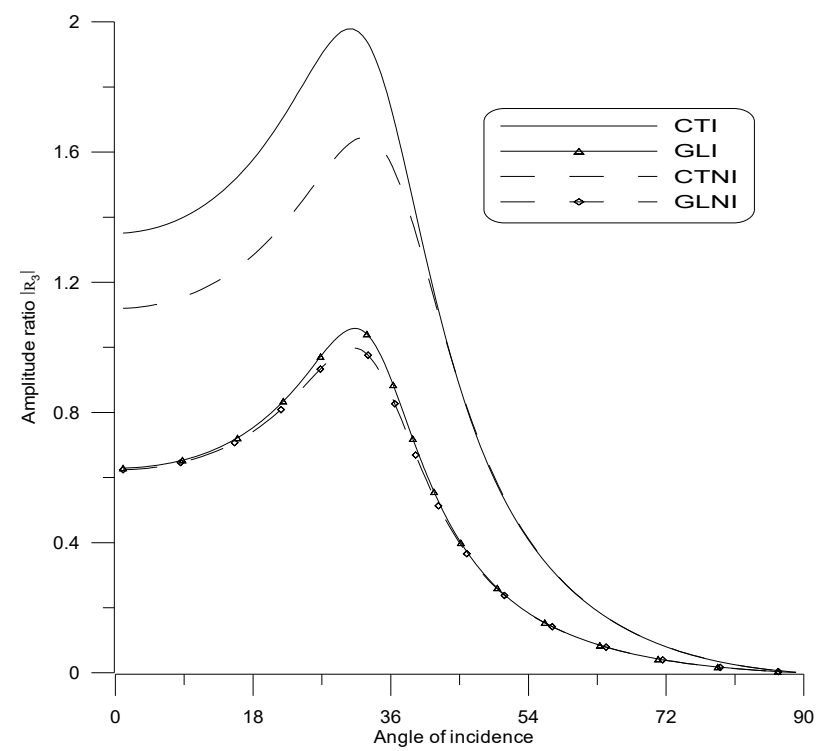

Fig.8. Variation of the amplitude ratio $\left|\mathrm{R}_{3}\right|$ for T-Wave.

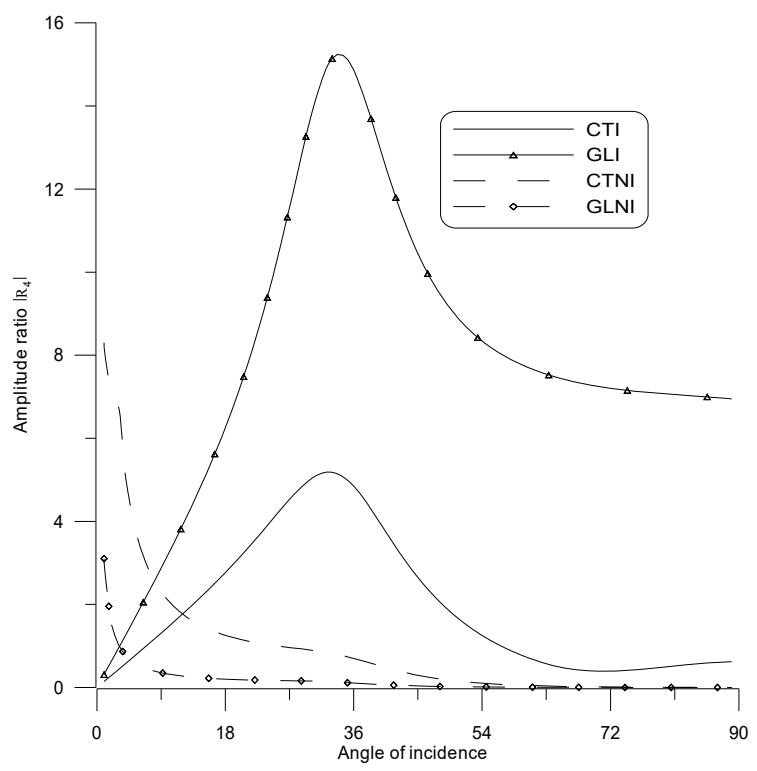

Fig.9. Variation of the amplitude ratio $\left|\mathrm{R}_{4}\right|$ for T-Wave.

\subsection{Incident MD-Wave}

Figure 10 is a plot of the amplitude ratio $\left|R_{l}\right|$. It is noticed that trends of curves for CTI are similar to those observed for CTNI with a significant difference in their values, which reveals the impact of impedance parameters. The amplitude ratio $\left|R_{l}\right|$ for GLI and GLNI is smaller that those observed for CTI and CTNI, which reveals the impact of relaxation time.

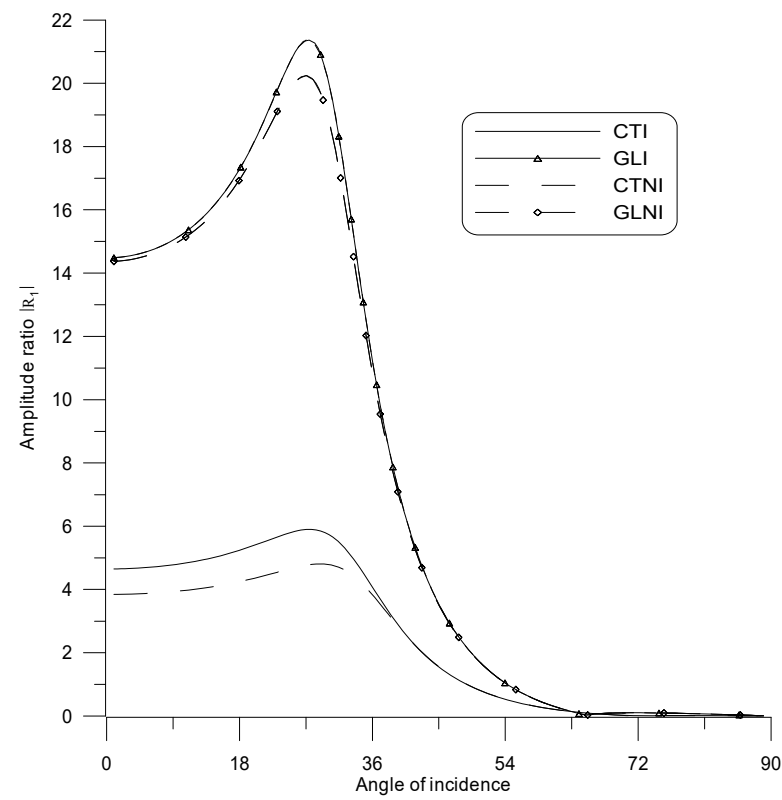

Fig.10. Variation of the amplitude ratio $\left|\mathrm{R}_{1}\right|$ for MD-Wave.

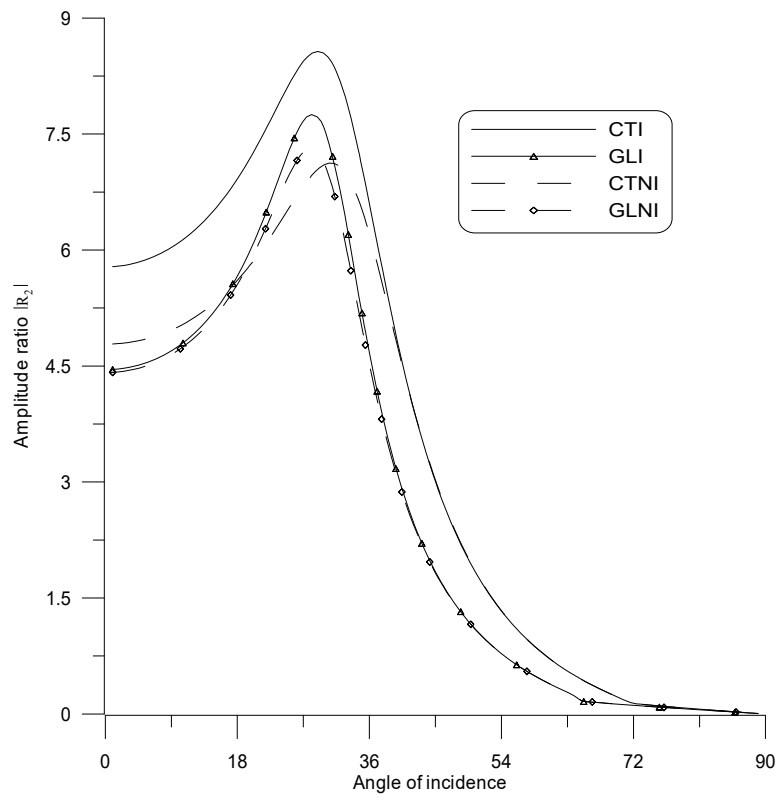

Fig.11. Variation of the amplitude ratio $\left|\mathrm{R}_{2}\right|$ for MD-Wave. 
Figure 11 shows the variations of $\left|R_{2}\right|$ for the CT-theory and GL-theory. It is observed that in both the cases the impedance parameter increases in the range $0^{0} \leq \theta_{0} \leq 27^{0}$ and vice-versa in the remaining range which ultimately approaches zero. It is noticed that the difference between the amplitude ratio for GLI and GLNI is smaller than that noticed for CTI and CTNI.

Figure 12 is the variation of the amplitude ratio $\left|R_{3}\right|$ along with the angle of incidence. It is observed that the trend of variation of amplitude ratio $\left|R_{3}\right|$ is similar to that observed for $\left|R_{2}\right|$ with a significant difference in their values.

Figure 13 shows the variations of $\left|R_{4}\right|$. It is noticed that initially there is a gradual increase for CTI and GLI i.e. in case of the impedance parameter whereas the values decrease for CTNI and GLNI i.e. in case of the non-impedance parameter in the range $0^{0} \leq \theta_{0} \leq 32^{\circ}$ and with the increase in $\theta_{0}$ the trend changes and the values tend towards the origin for the latter case.

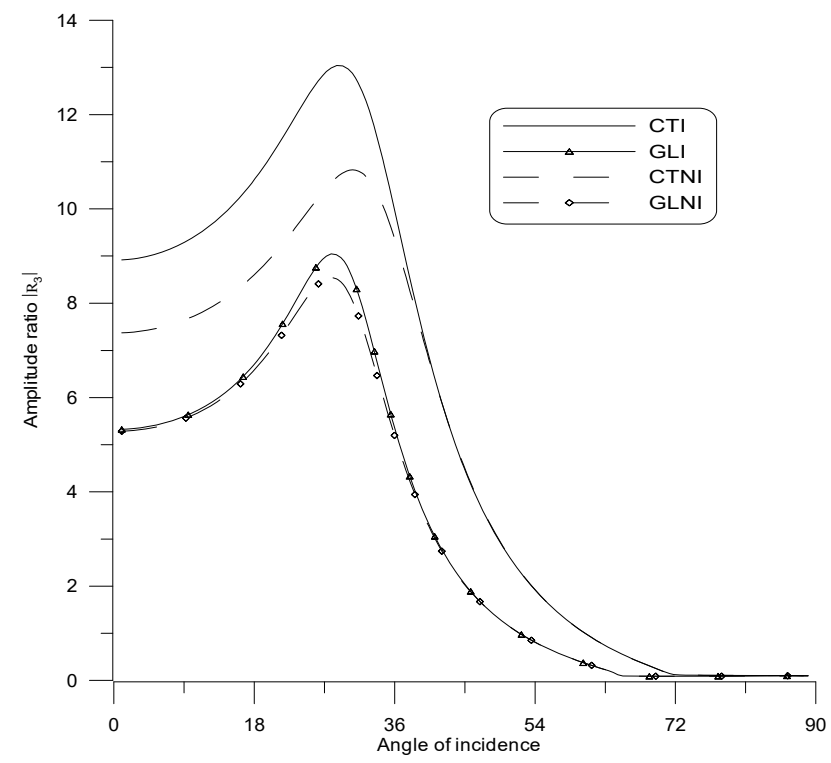

Fig.12. Variation of the amplitude ratio $\left|\mathrm{R}_{3}\right|$ for MD-Wave.

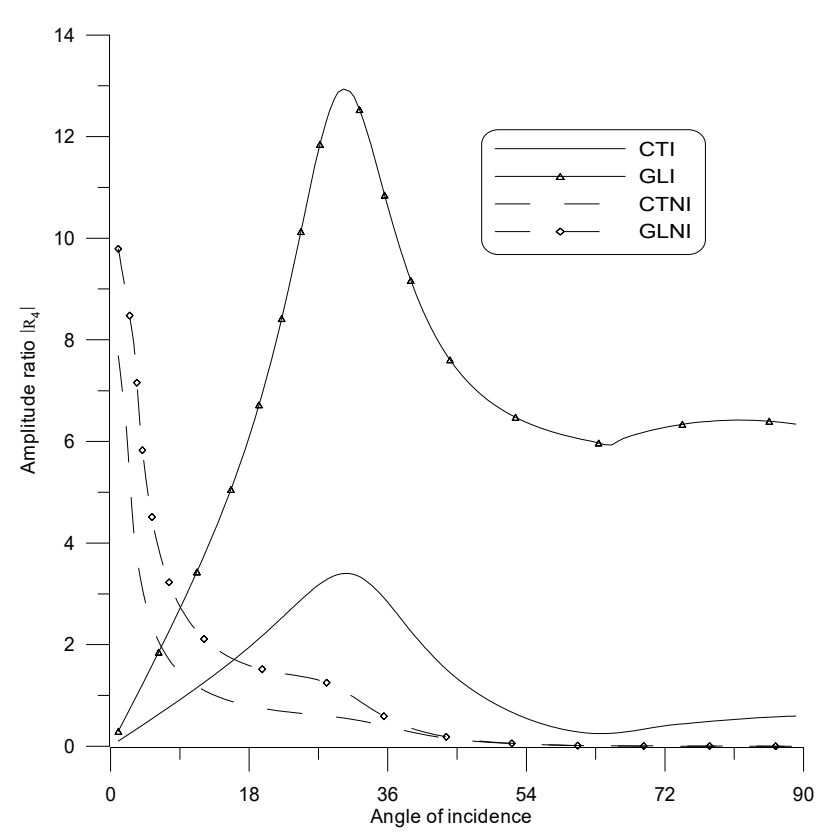

Fig.13. Variation of the amplitude ratio $\left|\mathrm{R}_{4}\right|$ for MD-Wave.

\section{Conclusion}

The problem of reflection for the impedance boundary condition is studied for a generalized thermoelastic diffusion medium in two relaxation theories of thermoelasticity. The following observations can be made:

(i) In case of P-Wave, the values of the amplitude ratio $\left|R_{1}\right|,\left|R_{2}\right|$ and $\left|R_{3}\right|$ for the case of the G-L theory are greater in the entire range as compared to those obtained for the $\mathrm{CT}$ theory of thermoelasticity, whereas in the absence of impedance parameter the values for the amplitude ratio $\left|R_{4}\right|$ show a decreasing trend and for the impedance parameter, it is shows an increasing trend in the entire range.

(ii) It is observed that the values of the amplitude ratio $\left|R_{1}\right|,\left|R_{2}\right|$ and $\left|R_{3}\right|$ for the case of T-Wave follows an opposite behaviour as observed for P-Wave i.e. the values of amplitude ratios for $\left|R_{1}\right|,\left|R_{2}\right|$ and $\left|R_{3}\right|$ are 
greater in case of the CT theory. In the presence of the impedance parameter the values of $\left|R_{4}\right|$ are greater in comparison to those observed for non-impedance parameter.

(iii) It is observed that trends of $\left|R_{1}\right|,\left|R_{2}\right|$ and $\left|R_{3}\right|$ are similar in nature for impedance and non-impedance parameter, whereas an opposite behaviour is observed for the case of $\left|R_{4}\right|$ in case of MD-Wave.

It is concluded that diffusion and relaxation times have a significant impact on reflection. Also, impedance parameters plays a vital role in reflection. The present new model is useful for more realistic models of thermo-diffusion elastic solids present in the earth's interior. Therefore, the problem is of geophysical interest, particularly for investigations concerned with an earthquake and other phenomena in seismology and engineering.

\section{Nomenclature}

$$
\begin{aligned}
a, b, D & \text {-thermodiffusion constants } \\
C & \text { - concentration } \\
K^{*} & \text { - thermal conductivity } \\
T & \text { - temperature distribution } \\
T_{0} & \text { - reference temperature } \\
t & \text { - time } \\
t_{i j} & - \text { component of stress tensor } \\
\vec{u} & \text { - displacement vector } \\
\alpha_{t} & - \text { coefficient of linear thermal expansion } \\
\delta_{i j} & - \text { Kronecker delta } \\
\lambda, \mu & \text { - Lame's constants } \\
\rho, C^{*} & \text { - density and specific heat respectively } \\
\tau_{0}, \tau_{l} & \text { - thermal relaxation times } \\
\tau^{0}, \tau^{l} & \text { - diffusion relaxation times }
\end{aligned}
$$

\section{Appendix-I}

$$
\begin{aligned}
& a_{1}=\frac{\mu}{\rho c_{1}^{2}}, \quad a_{2}=\frac{\rho c_{1}^{2}}{\beta_{1} T_{0}}, \quad a_{3}=\frac{\beta_{1}^{3} T_{0}^{2}}{\rho c_{1}^{2} \omega_{1} K^{*}}, \quad a_{4}=\frac{a \beta_{1} T_{0} c_{I}^{2}}{\beta_{2} K^{*} \omega_{1}}, \\
& a_{5}=\frac{D \beta_{1} \beta_{2} T_{0} \omega_{1}^{2}}{\rho c_{1}^{4}}, \quad a_{6}=\frac{D a \rho \omega_{1}^{2}}{\beta_{1}}, \quad a_{7}=\frac{\rho \omega c_{1}^{2}}{\beta_{2}}, \quad a_{8}=\frac{D b \rho \omega_{1}^{2}}{\beta_{2}} .
\end{aligned}
$$

\section{Appendix-II}

$$
\begin{aligned}
& F=\frac{a_{2} a_{3} a_{7} \tau_{e}+\left(1+a_{1}\right) a_{7} \tau_{g}+a_{4} a_{6} \tau_{e} \tau_{h} \omega^{2}-a_{8} \tau_{f} \tau_{g} \omega^{2}+a_{7}}{a_{7} \tau_{g}}, \\
& H=\frac{a_{2} a_{5} \tau_{f} \omega^{2}-\left(1+a_{1}\right) a_{8} \tau_{f} \omega^{2} k^{2}}{a_{7} \tau_{g} \tau_{h}},
\end{aligned}
$$




$$
\begin{aligned}
& G=\frac{a_{4}\left[\left(1+a_{1}\right) a_{6}+a_{5}\right] \tau_{e} \tau_{h} \omega^{2}+\mathrm{a}_{2} \mathrm{a}_{3}\left(a_{6}+a_{8}\right) \tau_{e} \tau_{\mathrm{f}} \omega^{2}+G_{1}}{a_{7} \tau_{g}}, \\
& G_{1}=\left(1+a_{7}\right) a_{7}-\left(1+a_{1}\right) a_{8} \tau_{f} \tau_{g} \omega^{2}-a_{2} a_{5} \tau_{f} \tau_{g}-a_{8} \tau_{f} \omega^{2}, \\
& \tau_{e}=\tau_{1}-\frac{\imath}{\omega}, \tau_{f}=\tau^{1}-\frac{\imath}{\omega}, \tau_{g}=\tau_{0}-\frac{\imath}{\omega}, \tau_{h}=\tau^{0}-\frac{\imath}{\omega} .
\end{aligned}
$$

\section{Appendix-III}

$$
\begin{aligned}
& d_{l}=\frac{\left[a_{8}\left(1+a_{1}\right)-a_{2} a_{5}\right] i \omega \tau_{f} \kappa_{l}^{4}+\left[a_{7}\left(1+a_{1}\right)-a_{8} \tau_{f} \omega^{2}\right] i \omega \kappa_{l}^{2}-a_{7} l \omega^{3}}{a_{2} a_{7} \tau_{e} \omega^{2}+a_{2}\left(a_{6}+a_{8}\right) \omega^{2} \tau_{e} \tau_{f} \kappa_{l}^{2}}, \\
& f_{l}=\frac{\left[a_{2} a_{5} \tau_{f}+a_{6}\left(1+a_{1}\right) \tau_{e}\right] i \omega \kappa_{l}^{4}-\left[a_{6} \tau_{d} i \omega^{3}\right] \kappa_{l}^{2}}{a_{2} a_{7} \tau_{e} \omega^{2}+a_{2}\left(a_{6}+a_{8}\right) \omega^{2} \tau_{e} \tau_{f} \kappa_{l}^{2}}, \quad \text { where }(l=1,2,3) .
\end{aligned}
$$

\section{Appendix-IV}

$$
\begin{aligned}
& a_{1 j}=-2 a_{1} \kappa_{j}^{2}\left(\frac{v_{j}}{v_{0}}\right)\left[\left(\frac{v_{0}}{v_{j}}\right)^{2}-\sin ^{2} \theta_{0}\right]^{\frac{1}{2}}-a_{9} \kappa_{j}^{2}-a_{2}\left(1+\tau_{l} i \omega\right) d_{j}-A_{j}, \\
& a_{14}=-2 a_{1} \kappa_{4}^{2}\left(\frac{v_{4}}{v_{0}}\right)^{2}\left[\left(\frac{v_{0}}{v_{4}}\right)^{2}-\sin ^{2} \theta_{0}\right]^{\frac{1}{2}}+\imath \omega Z_{1} \kappa_{4}\left(\frac{v_{4}}{v_{0}}\right)^{2} \sin \theta_{0}, \\
& a_{2 j}=-2 a_{1} \kappa_{j}^{2}\left(\frac{v_{j}}{v_{0}}\right)\left[\left(\frac{v_{0}}{v_{j}}\right)^{2}-\sin ^{2} \theta_{0}\right]^{\frac{1}{2}}+i \omega Z_{2} \kappa_{j}\left(\frac{v_{0}}{v_{j}}\right) \sin ^{2} \theta_{0}, \\
& a_{24}=-a_{1} \kappa_{4}^{2}\left(\frac{v_{4}}{v_{0}}\right)^{2} \sin ^{2} \theta_{0}-1 \omega Z_{2} \kappa_{4}\left(\frac{v_{4}}{v_{0}}\right)\left[\left(\frac{v_{0}}{v_{4}}\right)^{2}-\sin ^{2} \theta_{0}\right]^{\frac{1}{2}}, \\
& a_{3 j}=d_{j} \mathrm{i \kappa}\left(\frac{v_{j}}{v_{0}}\right)\left[\left(\frac{v_{0}}{v_{j}}\right)^{2}-\sin ^{2} \theta_{0}\right]^{\frac{1}{2}}, \quad a_{34}=0, \\
& a_{4 j}=f_{j} \kappa_{j}\left(\frac{v_{j}}{v_{0}}\right)\left[\left(\frac{v_{0}}{v_{j}}\right)^{2}-\sin ^{2} \theta_{0}\right]^{\frac{1}{2}} \\
& a_{44}=0, \quad A_{j}=a_{2}\left(1+\tau^{l} i \omega\right) f_{j}+1 \omega Z_{1} \kappa_{j}\left[\left(\frac{v_{0}}{v_{j}}\right)^{2}-\sin ^{2} \theta_{0}\right]^{\frac{1}{2}} \quad j=1-3 .
\end{aligned}
$$




\section{References}

[1] Nowacki W. (1974a): Dynamical problems of thermo diffusion in solids I.- Bull Acad. Pol. Sci. Ser. Sci. Tech, vol.22, pp.55-64.

[2] Nowacki W. (1974b): Dynamical problems of thermo diffusion in solids II.- Bull Acad. Pol. Sci. Ser. Sci. Tech, vol.22, pp.129-135.

[3] Nowacki W. (1974c): Dynamical problems of thermo diffusion in solids III, Bull Acad. Pol. Sci. Ser. Sci. Tech, vol.22, pp.257-266.

[4] Nowacki W. (1976): Dynamical problems of thermo diffusion in solids.- Engg. Frac. Mech, vol.8, pp.261-266.

[5] Sherief H.H., Hamza F. and Saleh H. (2004): The theory of generalized thermoelastic diffusion.- International Journal Engineering Science, vol.42, pp.591-608.

[6] Sherief H.H. and Saleh H. (2004): A half-space problem in the theory of generalized thermoelastic diffusion.International Journal of Solids and Structures, vol.42, pp.591-608.

[7] Singh B. (2005): Reflection of P and SV Waves from free surface of an elastic solid with generalized thermodiffusion.J. Earth Syst. Sci, vol.114, No.2, pp.159-168.

[8] Palani G. and Abbas I.A. (2009): Free convection MHD flow with thermal radiation from an impulsively-started vertical plate.- Nonlinear Analysis: Modelling and Control, vol.14, No.1, pp.73-84.

[9] Ibrahim A.A, El-Amin M.F. and Salama A (2009): Effect of thermal dispersion on free convection in a fluid saturated porous medium.- International Journal of Heat and Fluid Flow, vol.30, No.2, pp.229-236.

[10] Aouadi M. (2009): Theory of generalized micropolar thermoelastic diffusion under Lord-Shulman model.- Journal of Thermal Stresses, vol.32, pp.932-942.

[11] Othman M.I.A., Atwa S.Y. and Farouk R.M. (2009): The effect of diffusion on two dimensional problem of generalized thermoelasticity with Green-Naghdi theory.- International Communications in Heat and Mass Transfer, vol.36, No.8, pp.857-864.

[12] Kumar R. and Kansal T. (2012): Plane waves and fundamental solution in the generalized theories of thermoelastic diffusion.- IJAMM, vol.8, No.4, pp.21-34.

[13] El-Naggar A.M., Kishka Z., Abd-Alla A.M, Abbas I.A, Abo-Dahab S.M and Elsagheer M. (2013): On the initial stress, magnetic field, voids and rotation effects on plane waves in generalized thermoelasticity.- Journal of Computational and Theoretical Nanoscience, vol.10, No.6, pp.1408-1417.

[14] Marin M, Othman M.I.A and Abbas I.A. (2015): An extension of the domain of influence theorem for generalized thermoelasticity of anisotropic material with voids.- Journal of Computational and Theoretical Nanoscience, vol.12, No.8, pp.1594-1598.

[15] Othman M.I.A. and Said S.M. (2018): Effects of diffusion and internal heat source on a two-temperature thermoelastic medium with three-phase-lag model.- Archives of Thermodynamics, vol.39, No.2, pp.15-39.

[16] Saeed T., Ibrahim A.A. and Marin M. (2020): A GL model on thermo-elastic interaction in a poroelastic material using finite element method.- Symmetry, vol.12, No.3, pp.488 (1-14).

[17] Tiersten H.F. (1969): Elastic surface waves guided by thin films.- Journal of Applied Physics, vol.40, pp.770-789.

[18] Malischewsky P.G. (1987): Surface Waves and Discontinuities.- Elsevier, Amsterdam.

[19] Vinh P.C. and Hue T.T. (2014): Rayleigh waves with impedance boundary conditions in anisotropic solids.- Wave Motion, vol.51, pp.1082-1092.

[20] Singh B. (2017): Reflection of elastic waves from plane surface of a half-space with impedance boundary conditions.- Geosciences Research, vol.2, No.4, pp.242-253.

[21] Green A.E. and Lindsay K.A. (1972): Thermoelasticity.- Journal of Elasticity, vol.2, pp.1-7.

[22] Schoenberg A.C. (1971): Transmission and reflection of plane waves at an elastic-viscoelastic interface.Geophysics J.R. Astr. Soc., vol.25, pp.35-47.

[23] Thomas L. (1980): Fundamental of Heat Transfer.- Prentice Hall Inc., Englewmd Cliffs, NJ.

Received: June 5, 2021

Revised: September 27, 2021 